\title{
Alginate nanoparticles as non-toxic delivery system for miltefosine in the treatment of candidiasis and cryptococcosis
}

This article was published in the following Dove Press journal: International Journal of Nanomedicine

\author{
Cristina de Castro Spadari ${ }^{1} *$ \\ Fernanda Walt Mendes da Silva \\ de Bastiani ${ }^{1} *$ \\ Luciana Biagini Lopes ${ }^{2}$ \\ Kelly Ishida ${ }^{\prime}$
}

\begin{abstract}
'Laboratory of Antifungal Chemotherapy, Department of Microbiology, Institute of Biomedical Sciences, University of São Paulo, São Paulo, Brazil; ' ${ }^{2}$ Laboratory of Nanomedicine and Drug Delivery Systems, Department of Pharmacology, Institute of Biomedical Sciences, University of São Paulo, São Paulo, Brazil

*These authors contributed equally to this work
\end{abstract}

Introduction and objective: Previous studies indicate that miltefosine (MFS) may be an alternative as an antifungal agent; however, it presents several adverse effects. Thus, the aim of this study was to produce miltefosine-loaded alginate nanoparticles (MFS.Alg) for toxicity reduction to be used as an alternative for the treatment of cryptococcosis and candidiasis.

Methods: Alginate nanoparticles were produced using the external emulsification/gelation method, and their physicochemical and morphological characteristics were analyzed. MFS encapsulation efficiency, release assay and toxicity on red blood cells and on Galleria mellonella larvae were assessed. The antifungal activity was evaluated using in vitro and in vivo larval models of G. mellonella infected with Candida albicans (SC5314 and IAL-40), Cryptococcus neoformans H99 and Cryptococcus gattii ATCC 56990. The treatment efficacy was evaluated by survival curve, colony forming unit (CFU) counting and histopathological analysis.

Results: MFS.Alg nanoparticles presented a mean size of $279.1 \pm 56.7 \mathrm{~nm}$, a polydispersity index of $0.42 \pm 0.15$ and a zeta potential of $-39.7 \pm 5.2 \mathrm{mV}$. The encapsulation efficiency of MFS was $81.70 \pm 6.64 \%$, and its release from the nanoparticles occurred in a sustained manner. MFS in alginate nanoparticles presented no hemolytic effect and no toxicity in G. mellonella larvae. Treatment with MFS.Alg extended the survival time of larvae infected with $C$. albicans and $C$. gattii. In addition, the fungal burden reduction was confirmed by CFU and histopathological data for all groups treated with $200 \mathrm{mg} / \mathrm{Kg}$ of MFS.Alg.

Conclusion: These results support the use of alginate-based drug delivery systems as carriers for MFS for drug toxicity reduction and control of the fungal infection in the in vivo model of $G$. mellonella.

Keywords: nanocarriers, antifungal, drug delivery, Galleria mellonella, invasive fungal infection

\section{Introduction}

Infections caused by fungi have become a major public health problem and have been growing in number and severity in recent decades. ${ }^{1,2}$ Over $90 \%$ of all fungal-related deaths result from invasive fungal infections (IFIs) by Candida, Cryptococcus, Aspergillus and Pneumocystis species. ${ }^{3,4}$ Currently, five major antifungal drug classes are available to treat IFIs: azoles, echinocandins, polyenes, allylamines and pyrimidine analogues; 5,6 however, their use is limited by poor selectivity, high toxicity and resistance. ${ }^{7,8}$ Thus, the need for new treatment options for IFIs is evident.

Miltefosine (MFS) is an alkylphosphocholine analogue synthesized and evaluated in the 1980's for antineoplastic activity. ${ }^{9}$ Currently, oral administration of MFS is
Correspondence: Kelly Ishida Laboratory of Antifungal Chemotherapy, Department of Microbiology, Institute of Biomedical Sciences, University of São Paulo, Prof. Lineu Prestes Avenue, | 374 - 05508-000, ICB |I, Lab |50, São Paulo, SP, Brazil

Tel +55(I I)309l7204

Email ishidakelly@usp.br 
recommended for visceral and cutaneous leishmaniasis treatments, whereas its topical administration is approved for management of skin metastases of breast cancer. ${ }^{9-12}$ In addition, MFS has considerable antifungal potential in vitro against a wide range of pathogenic fungi, including Candida spp., Cryptococcus spp., Aspergillus spp., Fusarium spp., Scedosporium spp., Sporothrix spp., Paracoccidioides spp., Histoplasma capsulatum, Coccidioides posadasii, and dermatophytes, ${ }^{13-18}$ and thus, could represent a potential alternative to the available drugs for treatment of IFIs.

However, in spite of its demonstrated antifungal activity, MFS presents pharmacokinetic disadvantages and systemic toxic effects that restrict its clinical use for treatment of IFIs. Serious gastrointestinal effects (such as nausea, vomiting, and diarrhea) related to oral administration of MFS have been reported, and its detergent properties may contribute to damage of the gastrointestinal mucosa. ${ }^{19,20}$ Other frequently observed MFS-related toxic effects are mainly associated with the kidneys and liver, in addition to teratogenicity and high hemolytic activity. ${ }^{19}$ In attempt to reduce its toxicological effects and to expand its clinical use (to include treatment of IFIs), encapsulation of MFS in nanocarriers is proposed here.

Among the various drug carriers employed to improve drug delivery, there are polymeric nanoparticles, such as those produced with alginate. Alginate is a natural, nontoxic, biocompatible and non-immunogenic polymer that presents biodegradable and mucoadhesive properties, a low cost of production, and is readily available. ${ }^{21,22}$ Other antifungal agents, such as azoles and polyenes, have been incorporated into alginate-based carriers, and have showed promising in vitro and in vivo results in terms of antifungal activity, improvement of bioavailability, and dose reduction and systemic toxicity. ${ }^{23}$ No previous study has attempted to encapsulate MFS in alginate-based nanocarriers to assess its efficacy for treatment of IFIs.

In view of the above, the present study aims at developing alginate nanocarriers for MFS encapsulation to overcome the unfavorable toxicity profile, and to serve as an alternative treatment for cryptococcosis and candidiasis.

\section{Materials and methods}

\section{Materials}

Sodium alginate (MP Biomedicals, Solon, OH, USA), sunflower oil, sorbitan monooleate (SPAN 80, Sigma Aldrich, St. Louis, MO, USA), poloxamer 407 (Sigma Aldrich, St. Louis, MO, USA), isopropanol (Merck,
Darmstadt, Germany), and calcium chloride (Synth, Diadema, SP, Brazil) were used for nanoparticle production. Miltefosine (MFS, Cayman Chemical, Ann Arbor, MI, USA), amphotericin B (AMB, Sigma Aldrich, St. Louis, MO, USA) and fluconazole (FLC, Sigma Aldrich, Laramie, WY, USA) were used as standard drugs.

\section{Fungal collection}

Clinical ("IAL") and standard strains of Candida spp. [C. albicans (SC5314 and IAL-40), C. parapsilosis (ATCC 22019 and IAL-17), C. tropicalis (ATCC 200956 and IAL-01), C. glabrata (ATCC 2001 and IAL-23) and C. krusei (ATCC 6258 and IAL-30)] and standard strains of Cryptococcus spp. [C. neoformans stricto sensu ATCC 208821 (or H99), C. neoformans CAP59 (H99 acapsular mutant), and C. gattii lato sensu ATCC 56990] were used in this work.

The strains were stored in Brain and Heart Infusion (BHI) medium and $20 \%$ glycerol at $-80{ }^{\circ} \mathrm{C}$, recovered in Sabouraud dextrose medium, and kept in Sabouraud agar at $4{ }^{\circ} \mathrm{C}$ during the tests. Before each assay, the yeasts were twice subcultured in Sabouraud dextrose medium for $48-72 \mathrm{~h}$ at $35^{\circ} \mathrm{C}$.

\section{Nanoparticle production}

The alginate nanoparticles (Nano.Alg) were obtained by emulsification using the external gelation method. First, $1.35 \mathrm{~g}$ of $1 \%$ alginate aqueous solution was mixed with $2.04 \mathrm{~g}$ of sunflower oil containing 3\% SPAN 80 and homogenized for $1 \mathrm{~min}$ using a hand-held homogenizer for emulsification. The emulsion was sonicated for $10 \mathrm{~min}$ in pulses (50 s on, $10 \mathrm{~s}$ off) in an ice bath using 40\% maximum amplitude (VCX500, Sonics, Newtown, CT, USA). While stirring, $1 \mathrm{~mL}$ of $0.22 \mathrm{M}$ calcium chloride solution with $0.5 \%$ poloxamer 407 was added dropwise. The mixture was sonicated for $5 \mathrm{~min}$ in pulses (50 s on, $10 \mathrm{~s}$ off), followed by 30 min of magnetic stirring. After these steps, the system was centrifuged for $5 \mathrm{~min}$ at $3000 \mathrm{~g}$, the supernatant was removed and isopropanol (P.A.) was added to remove oil residues. Subsequently, $500 \mu \mathrm{L}$ of $10 \%$ trehalose was added prior to freeze-drying for $24 \mathrm{~h}$ (FreeZone 2.5 freeze-dryer, Labconco, Kansas, MO, USA) to obtain a homogeneous fine powder of alginate nanoparticles.

MFS-loaded alginate nanoparticles (MFS.Alg) were obtained using the same protocol, except that MFS $(3 \mathrm{mg})$ was added to the alginate aqueous dispersion. ${ }^{24}$ 


\section{Physicochemical and morphological} characterization of the nanoparticles Measurement of particle size, size distribution, and zeta potential

The unloaded and MSF-loaded alginate nanoparticles (Nano. Alg and MFS.Alg, respectively) were diluted (1:100, v/v) in distilled water before and after freeze-drying for determination of the hydrodynamic diameter $(\mathrm{Dz})$ and size distribution (Pdi) by Dynamic Light Scattering (DLS, Zetasizer NanoZS90, Malvern Instruments, Worcestershire, UK). Zeta potential was measured by electrophoresis after sample dilution in distilled water $(1: 100, \mathrm{v} / \mathrm{v}$, Zetasizer NanoZS 90 , Malvern Instruments, Worcestershire, UK).

\section{Electron microscopy}

Transmission electron microscopy (TEM) and scanning electron microscopy (SEM) were performed to assess the morphological characteristics of Nano.Alg and MFS.Alg. For TEM, one drop of the sample was placed on a 300 mesh copper grid covered with FormVar film, contrasted with $2 \%$ phosphotungstic acid for $1 \mathrm{~min}$ and observed on the transmission electron microscope (Tecnai G20, FEI, Thermo Scientific, Hillsboro, Oregon, USA). For SEM, the glass coverslips were covered with poly-L-lysine for $30 \mathrm{~min}$ and rinsed before placing the samples on their surface. The samples were left at room temperature for $24 \mathrm{~h}$ for drying and metallized with platinum $(10 \mathrm{~nm})$ before analysis on the scanning electron microscope (Quanta 650 FEG, FEI, Thermo Scientific, Hillsboro, Oregon, USA).

\section{Fourier transform infrared spectroscopy (FTIR)} Spectroscopic analyzes in the infrared region were conducted to verify interactions between MFS and formulation components, and compared with free MFS and Nano. Alg, FTIR spectra were obtained using $\mathrm{KBr}$ pellets in a range from 4500 to $500 \mathrm{~cm}^{-1}$, with a resolution of $2 \mathrm{~cm}^{-1}$ (Shimadzu IR Prestige-21, Kyoto, Japan).

\section{Miltefosine encapsulation percentage and in vitro release assay}

The efficiency of MFS encapsulation in alginate nanoparticles was evaluated using the formula: 100- (MFS in supernatant $\times 100$ /amount of initial MFS). ${ }^{25}$ MFS was quantified in the supernatant by a colorimetric method based on the complexation of MFS with ammonium ferrotiocianate, and the resulting complex was detected at $460 \mathrm{~nm}$ (Epoch 2, BioTek, Winooski, VT, USA). ${ }^{26}$ Calibration curves for MFS in distilled water $(15.62-2000 \mu \mathrm{g} / \mathrm{mL})$ were prepared to determine drug concentration in the samples. The experiment was performed three times.

To verify whether MFS was released from the nanoparticles, the nanoparticle powder was suspended in $1 \mathrm{~mL}$ of sterile water and incubated at $37{ }^{\circ} \mathrm{C}$ while stirring at $200 \mathrm{rpm}$. At 6,12 and $24 \mathrm{~h}$, the samples were centrifuged for $5 \mathrm{~min}$ at $3000 \mathrm{~g}$ and the supernatant was collected for MFS quantification by the colorimetric method described above. Nano.Alg was used as a negative control and three independent experiments were performed. The supernatant was also analyzed by dynamic light scattering, and the absence of populations with the same characteristics of the nanoparticles indicated their sedimentation.

\section{Toxicity assays}

Cytotoxicity on red blood cells (RBC)

A suspension of $4 \%$ erythrocytes from sheep's blood (Newprovi, Pinhais, Paraná, Brazil) (v/v in 5\% sterile glucose solution) was exposed to MFS in freeform or in alginate nanoparticles at $1-128 \mu \mathrm{g} / \mathrm{mL}$ for $2 \mathrm{~h}$ in a $37{ }^{\circ} \mathrm{C}$ bath. Negative (untreated) and positive $(0.1 \%$ triton $\mathrm{X}-100$ ) controls were also used in this test. After incubation, the samples were centrifuged at $1500 \mathrm{~g}$ for $10 \mathrm{~min}$ and the supernatant was analyzed at $540 \mathrm{~nm}$ (Epoch 2, BioTek, Winooski, VT, USA) to determine the hemolytic activity percentage (HA\%). ${ }^{27}$ Three independent experiments were performed.

\section{Toxicity on Galleria mellonella larvae}

Galleria mellonella caterpillars $(2.0-2.5 \mathrm{~cm}$ in length and 150-200 mg of body weight) were treated with free MFS $(25-200 \mathrm{mg} / \mathrm{kg}$ ) or MFS.Alg (100-200 mg/kg of MFS) by injecting $10 \mu \mathrm{L}$ of each treatment into the last pro-leg of the larvae ( $\mathrm{n}=18$ larvae/group). ${ }^{28}$ Alginate nanoparticles (Nano. Alg) and PBS were used as controls. The caterpillars were incubated at $37{ }^{\circ} \mathrm{C}$ and observed daily for 5 days to assess survival. Three independent experiments were performed.

\section{In vitro antifungal activity}

The minimum inhibitory concentrations (MIC) of antifungals were determined by the broth microdilution method for Candida and Cryptococcus. ${ }^{29}$ The antifungal drugs were serially diluted (1:2) in RPMI 1640 medium buffered with 0.16 M 3-morpholinopropane-1-sulfonic acid (MOPS, Sigma-Aldrich, St. Louis, MO, USA), $\mathrm{pH} 7.0$, in flatbottom 96-well microplates. Then, $100 \mu \mathrm{L}$ of the fungal suspension was added to each well for a final fungal concentration of $0.5-2.5 \times 10^{3} \mathrm{CFU} / \mathrm{mL}$, and final antifungal 
concentrations of $0.03-16 \mu \mathrm{g} / \mathrm{mL}$ for MFS and AMB, 0.12-$64 \mu \mathrm{g} / \mathrm{mL}$ for FLC, and $0.78-600 \mu \mathrm{g} / \mathrm{mL}$ for MFS in alginate nanoparticles (MFS.Alg). In parallel, Nano.Alg was diluted under the same conditions as MFS.Alg to evaluate the interference of the formulation components in the antifungal activity. Microplates were incubated in a humid chamber in the dark at $35{ }^{\circ} \mathrm{C}$ for $48 \mathrm{~h}$ (Candida spp.) or $72 \mathrm{~h}$ (Cryptococcus spp.), and the fungal growth inhibition was visually observed. The lowest drug concentration that inhibited $90 \%$ of fungal growth was defined as the MIC values for AMB, MFS and MFS.Alg, and the lowest drug concentration that inhibited $50 \%$ of the fungal growth was defined as the MIC values for FLC. Candida spp. strains were classified as resistant or susceptible or dose-dependent, according to criteria established by the M27-S4 document. ${ }^{30}$

The minimum fungicidal concentration (MFC) was determined by culturing a $10-\mu \mathrm{L}$ aliquot of the yeast suspension treated with the inhibitory concentrations of antifungals on drug-free Sabouraud dextrose agar at $35^{\circ}$ $\mathrm{C}$ for $48-72 \mathrm{~h}$. The MFC was determined as the lowest concentration that reduced $99.9 \%$ of fungal viability; the fungicidal effect was considered when the MFC value was up to four times the MIC value. ${ }^{31}$

\section{In vivo antifungal activity on the Galleria mellonella larval model}

Before initiating the treatment with antifungals, we evaluated the virulence of Candida and Cryptococcus strains on the larval model of G. mellonella. The caterpillars in the last larval stage $(2.0-2.5 \mathrm{~cm}$ in length and a body weight of $150-200 \mathrm{mg}$ ) were infected with $10 \mu \mathrm{L}$ of the fungal suspension in PBS $\left(5 \times 10^{7} \mathrm{CFU} / \mathrm{mL}\right.$ for Candida spp. and $5 \times 10^{8} \mathrm{CFU} / \mathrm{mL}$ for Cryptococcus spp.) in the last pro-leg and incubated at $37{ }^{\circ} \mathrm{C} .{ }^{28}$ All groups, including the PBS group, were monitored every day after inoculation for 5 or 7 days (Candida and Cryptococcus, respectively) to construct the survival curves ( $\mathrm{n}=18$ larvae/group). Following analysis, two Candida and two Cryptococcus strains were selected for the antifungal treatments.

For the evaluation of antifungal activity, the G. mellonella caterpillars were infected, as described above, with $C$. albicans (SC5314 and IAL-40), C. neoformans H99 and C. gattii ATCC 56990. Thirty minutes post-infection, antifungal treatments were initiated with the administration of $10 \mu \mathrm{L}$ of free MFS $(10,20$ and $40 \mathrm{mg} / \mathrm{Kg}$ ) and MFS.Alg (100 and $200 \mathrm{mg} / \mathrm{Kg}$ of MFS) and the larvae incubated at $37^{\circ} \mathrm{C}$. Caterpillars untreated (PBS group) and treated with Nano.Alg were included in the assay. The survival of the larvae was monitored every day for up to 5 or 7 days (Candida and Cryptococcus, respectively) for construction of the survival curves ( $\mathrm{n}=22$ larvae/group). Two independent experiments were performed.

In addition, fungal burden determination and histopathological analysis were performed in G. mellonella larvae. For this, on the second day post-infection, 6 larvae from each group were weighed and the tissue was macerated and homogenized in $1 \mathrm{~mL}$ of sterile PBS followed by serial dilutions, plated on Sabouraud dextrose agar containing chloramphenicol $(50 \mu \mathrm{g} / \mathrm{mL})$, and incubated at $37^{\circ} \mathrm{C}$ for $48 \mathrm{~h}$ to obtain the number of colony forming units (CFU) per gram of larvae (CFU/g). Concomitantly, 2 larvae from each group were fixed with $10 \%$ formaldehyde in PBS for preparation of the histological sections and stained with hematoxylin-eosin (HE). Histological sections were subjected to semi-quantification analysis under optical microscopy (DM750, Leica, São Paulo, Brazil), according to the criteria described by Quintella et al. ${ }^{32}$

\section{Statistical analyses}

The results are reported as mean and standard deviation, and the data were statistically analyzed using the Student's $t$-test and ANOVA, followed by Dunnett's post-hoc test. G. mellonella larvae survival data were analyzed using logRank test. The 95\% confidence level was considered significant for all analyses using GraphPad Prism (version 5.00 for Windows, GraphPad Software, San Diego, California, USA).

\section{Results}

\section{Characterization of the alginate nanoparticles}

Unloaded nanoparticles (Nano.Alg), obtained by the emulsification technique with external gelation, were negatively charged $(-36.2 \pm 6.8 \mathrm{mV})$, had a mean size of $346.5 \pm 30.6 \mathrm{~nm}$ and a Pdi of $0.40 \pm 0.07$; similar characteristics were observed for miltefosine-loaded alginate nanoparticles (MFS.Alg) $(P>0.05)$, suggesting that drug encapsulation did not modify the particles' characteristics (Table 1; Figure S1). Additionally, these characteristics were not pronouncedly affected by utilization of $10 \%$ trehalose as a cryoprotector for freeze-drying and PBS for powder reconstitution (Table 1).

As demonstrated by TEM and SEM analyses, alginate nanoparticles seemed well dispersed and homogenously distributed with a relatively spherical form and a regular surface, presenting a similar size to the particle mean obtained by DLS $(\sim 300 \mathrm{~nm})$. Moreover, MFS encapsulation did not affect the nanoparticle morphology (Figure 1). 
Table I Physicochemical characteristics of unloaded (Nano.Alg) and miltefosine-loaded (MFS.Alg) alginate nanoparticles before and after the freeze-drying process

\begin{tabular}{|l|l|l|l|}
\hline & Size $\mathbf{( n m )}$ & Pdi & Zeta potential (mV) \\
\hline Nano.Alg before freeze-drying & $335.7 \pm 10.9$ & $0.35 \pm 0.06$ & $-21.1 \pm 1.3$ \\
Nano.Alg after freeze-drying & $346.5 \pm 30.6$ & $0.40 \pm 0.07$ & $-36.2 \pm 6.8^{*}$ \\
MFS.Alg before freeze-drying & $320.2 \pm 37.9$ & $0.43 \pm 0.14$ & $-19.0 \pm 3.1$ \\
MFS.Alg after freeze-drying & $279.1 \pm 56.7$ & $0.42 \pm 0.15$ & $-39.7 \pm 5.2^{*}$ \\
\hline
\end{tabular}

Notes: Data represent mean \pm standard deviation. $* P<0.05$ compared with nanoparticles before freeze-drying (Student's $t$-test).

To assess drug-polymer interactions that might impact drug release, FITR analysis was performed. Free MFS IR spectra contained peaks at $2950-2850 \mathrm{~cm}^{-1}$ that correspond to $\mathrm{CH}_{2}$ stretching in the long hexadecyl-chain, at $1520-1490 \mathrm{~cm}^{-1}$ that correspond to $\mathrm{CH}_{2}$ bending, and at $1260-960 \mathrm{~cm}^{-1}$ that correspond to the phosphocholine group (Figure S2A), as previously described. ${ }^{26}$ Main MFS-related peaks were observed in the IR spectrum obtained from MFS.Alg, suggesting no peak shifts due to reactions and/or strong interactions of MFS with formulation components. In contrast, none of these peaks appeared in the Nano.Alg spectrum (Figure S2B-C).

\section{Miltefosine was encapsulated in alginate nanoparticles and presented sustained release in vitro}

The encapsulation efficiency of MFS in the alginate nanoparticles was $81.70 \% \pm 6.64$, and the drug was released in a sustained manner from the alginate-based nanoparticles: approximately $112.5 \mu \mathrm{g} / \mathrm{mL}$ (4.68\%) of the drug was released in the first $6 \mathrm{~h}$ (Figure 2), while $\sim 181.25 \mu \mathrm{g} / \mathrm{mL}$ (7.55\%) of the drug was released at the longest time point assessed (24 h).
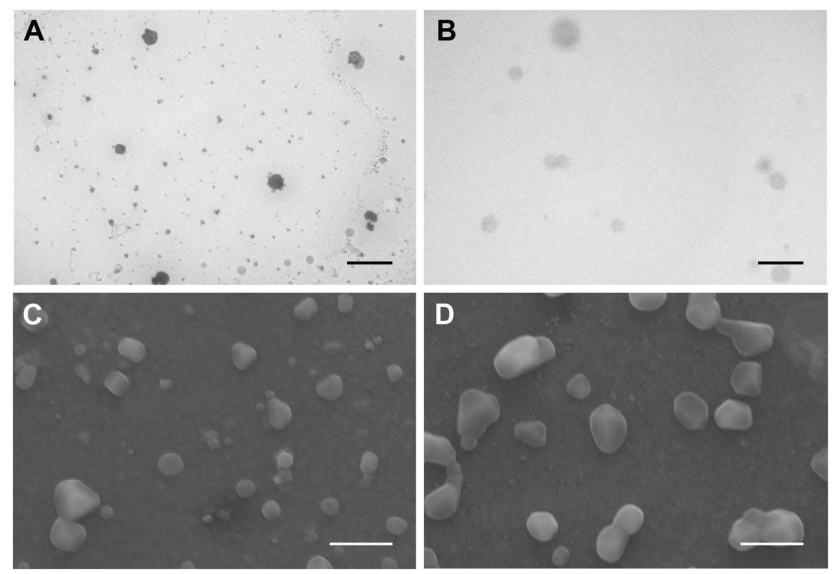

Figure I Transmission electron microscopy $(\mathbf{A}, \mathbf{B})$ and scanning electron microscopy (C, D) of alginate nanoparticles. Unloaded (Nano.Alg) (A, C) and miltefosine-loaded (MFS.Alg) (B, D) nanoparticles. Bars in (A) and (B): $2 \mu \mathrm{m}$; bars in (C) and (D): $0.5 \mu \mathrm{m}$.

\section{Miltefosine encapsulation in alginate nanoparticles reduced drug toxicity}

To assess the effect of MFS encapsulation on drug toxicity, hemolysis and survival of $G$. mellonella larvae were assessed as a function of drug concentration.

As a drug solution, MFS promoted hemolysis (50\%) at concentrations close to $35 \mu \mathrm{g} / \mathrm{mL}$, while no hemolytic effect was observed when the drug was incorporated into the alginate nanoparticles (MFS.Alg), even when its concentration was 3.65 -fold higher $(128 \mu \mathrm{g} / \mathrm{mL})$. Incubation with Nano.Alg (used as a control) did not result in hemolysis, suggesting that the nanocarrier itself is safe.

The toxicity of free MFS in G. mellonella larvae was observed at doses $\geq 50 \mathrm{mg} / \mathrm{kg}$, which resulted in larvae survival of $77.8 \%$ ( $P<0.05$ compared to PBS). In contrast, MFS.Alg at 100 and $200 \mathrm{mg} / \mathrm{kg}$ failed to increase mortality compared to PBS ( $P>0.05$; Figure 3$)$, demonstrating that MFS encapsulation in alginate-based nanocarriers protected the larvae from its toxic effects. In addition, unloaded alginate nanoparticles (Nano.Alg) did not lead to mortality of G. mellonella larvae (Figure 3).

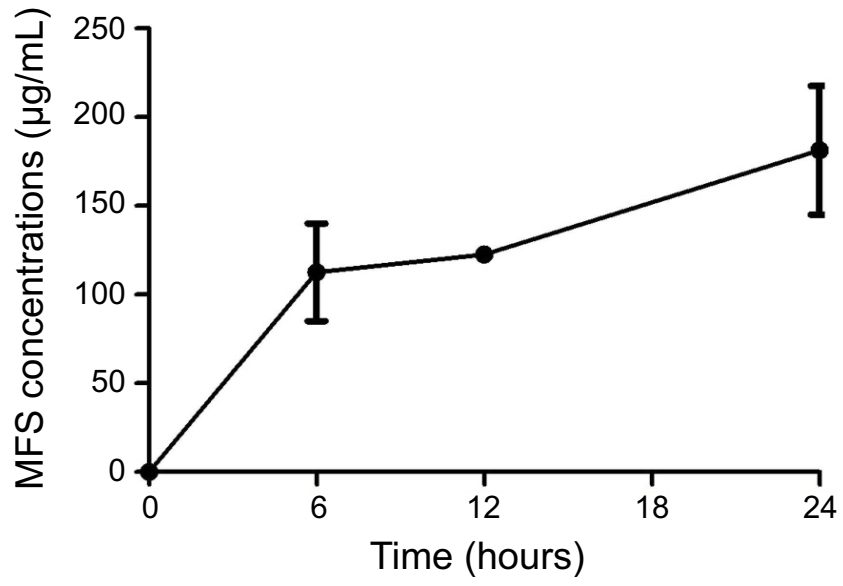

Figure 2 Release of miltefosine (MFS) from alginate nanoparticles. In vitro release at $37^{\circ} \mathrm{C}$, with constant agitation (200 rpm). 


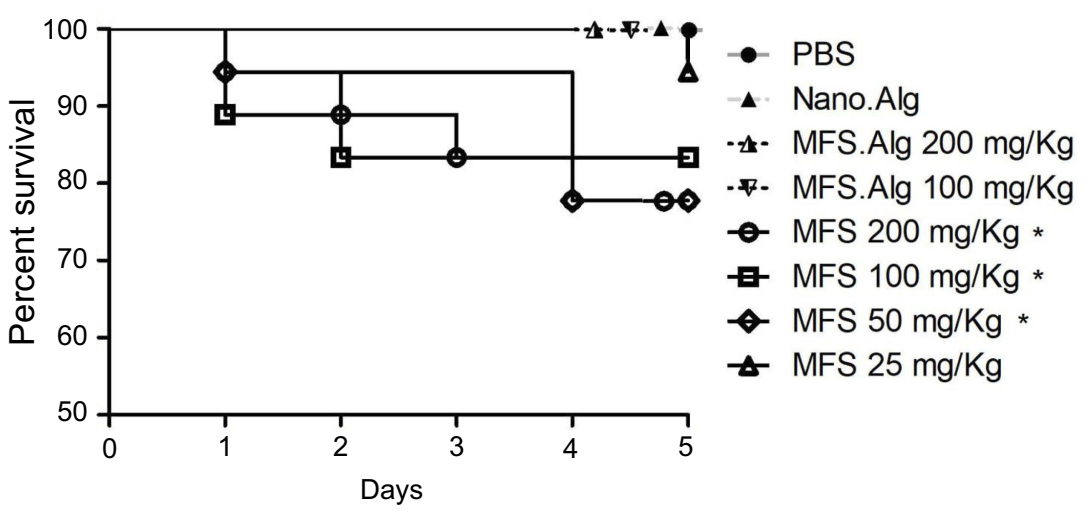

Figure 3 Toxicity of free miltefosine (MFS) and miltefosine-loaded alginate nanoparticles (MFS.Alg) in Galleria mellonella larvae. $* P<0.05$ when compared with untreated larvae (PBS group). Unloaded alginate nanoparticles (Nano.Alg) were also tested and no toxicity was observed.

\section{In vitro antifungal activity}

For antifungal susceptibility testing, we selected Cryptococcus spp. and FLC-resistant and FLCsusceptible Candida spp. strains (Table 2). Notably, MFS showed antifungal efficacy against FLC-resistant Candida spp. strains (Table 2). Free MFS inhibited fungal growth at concentrations from 0.5 to $2 \mu \mathrm{g} / \mathrm{mL}$ against Candida and Cryptococcus, a similar effect observed for AMB (from 0.03 to $2 \mu \mathrm{g} / \mathrm{mL}$ ). Both antifungals exhibited fungicidal effects on the pathogenic yeasts (Table 2).

Altogether, MFS in the alginate nanoparticles (MFS.Alg) showed a lower inhibitory effect than free MFS, except against the acapsular C. neoformans strain (CAP59) (Table 2). MIC values for MFS.Alg were 25-times higher when compared to free MFS on C. neoformans $\mathrm{H} 99$ and C. gattii ATCC 56990 (Table 2); however, for Candida spp., MFS.Alg showed the lowest inhibitory activity (300-600 $\mu \mathrm{g} / \mathrm{mL}$; up to 600 times) (Table 2). Nano.Alg was evaluated as a control, and no inhibitory effect on yeast was observed (data not shown).

\section{In vivo antifungal efficacy}

The virulence assay of Candida and Cryptococcus strains on G. mellonella larvae was carried out to select the fungal strains for antifungal treatments (Figure S3). Among Candida species, C. albicans SC5314 and IAL-40 were the most virulent strains, followed by $C$. tropicalis IAL01, C. krusei ATCC 6258 and C. parapsilosis IAL-17 (Figure S3A). Both C. neoformans $\mathrm{H} 99$ and C. gattii ATCC 56990 were virulent when compared with the PBS group $(P<0.0001$; Figure S3B $)$. Thus, $C$. albicans SC5314 (FLC-susceptible), C. albicans IAL-40 (FLC-resistant), C. neoformans $\mathrm{H} 99$ and C. gattii ATCC 56990 were selected for infection of G. mellonella larvae and assessment of antifungal efficacy of the nanoparticles.
Treatment of G. mellonella larvae infected with C. albicans SC5314 or IAL-40, or C. gattii ATCC 56990 using either free MFS or MFS.Alg significantly extended larvae survival time, except in the larvae infected with $C$. neoformans H99 (Figure 4). In all doses tested, free MFS and MFS.Alg increased the survival of larvae infected with $C$. albicans SC5314 by $75 \%$ when compared to the untreated group $(P<0.0001)$. The survival of larvae infected with $C$. albicans IAL-40 (FLC-resistant) was improved only by treatments with $20 \mathrm{mg} / \mathrm{Kg}$ of free MFS $(P=0.0007)$ and $200 \mathrm{mg} / \mathrm{Kg}$ of MFS. $\operatorname{Alg}(P=0.0022)$. Treatments with 100 and $200 \mathrm{mg} / \mathrm{Kg}$ of MFS. Alg led to $80 \%$ survival in larvae infected with $C$. gattii ATCC $56990(P<0.0001)$, while free MFS at doses of $\geq 20 \mathrm{mg} / \mathrm{Kg}$ also increased larvae survival $(P<0.001)$ (Figure 4$)$.

In addition to the survival curve, the fungal burden was evaluated on the $2^{\text {nd }}$ day after antifungal treatment (Figure 5). MFS.Alg at $200 \mathrm{mg} / \mathrm{Kg}$ led to a significant decrease in fungal burden for all Candida spp. and Cryptococcus spp. strains, while at $100 \mathrm{mg} / \mathrm{Kg}$, MFS.Alg decreased fungal burden only in larvae infected with $C$. albicans SC5314 $(P<0.001)$. Free MFS at 10 and $20 \mathrm{mg} / \mathrm{Kg}$ was effective at decreasing fungal burden of $C$. albicans SC5314 $(P<0.05)$, whereas $C$. albicans IAL-40 was reduced only in larvae treated with $10 \mathrm{mg} / \mathrm{Kg}$ of free MFS $(P<0.05)$. Although free MFS increased larvae survival after the $5^{\text {th }}$ day of infection with $C$. gattii ATCC 56990, the fungal burden on the $2^{\text {nd }}$ day post-infection was similar to the untreated group; in contrast, free MFS at 20 and $40 \mathrm{mg} / \mathrm{Kg}$ led to a significant reduction of fungal burden of $C$. neoformans $\mathrm{H} 99$ on the $2^{\text {nd }}$ day post-infection $(P<0.05)$ (Figure 5 ).

The fungal burden reduction was also observed by histopathological analysis and corroborated with CFU/g data after treatments with MFS.Alg and free MFS (Figure 5, Table S1). Beyond fungal burden reduction, both MFS.Alg doses reduced Cryptococcus dissemination in the larval tissue and C. albicans 
Table 2 Susceptibility of Candida spp. and Cryptococcus spp. strains to fluconazole (FLC), amphotericin B (AMB), free miltefosine (MFS) and miltefosine-loaded alginate nanoparticles (MFS.Alg)

\begin{tabular}{|c|c|c|c|c|c|c|c|c|}
\hline \multirow[t]{3}{*}{ Strains } & \multicolumn{8}{|c|}{ Concentrations $(\mu \mathrm{g} / \mathrm{mL})$} \\
\hline & \multicolumn{2}{|l|}{$\mathrm{FLC}^{\mathrm{a}}$} & \multicolumn{2}{|c|}{$\mathrm{AMB}^{\mathrm{a}}$} & \multicolumn{2}{|l|}{ MFS } & \multicolumn{2}{|c|}{ MFS.Alg } \\
\hline & MIC & MFC & MIC & MFC & MIC & MFC & MIC & MFC \\
\hline $\begin{array}{l}\text { C. albicans } \\
\text { SC5314 }\end{array}$ & 2 & 4 & 0.12 & 0.12 & 1 & I & 300 & 600 \\
\hline $\begin{array}{l}\text { C. albicans } \\
\text { IAL-40 }\end{array}$ & $64^{\mathrm{R}}$ & $>64$ & 0.12 & 0.12 & I & I & 600 & $>600$ \\
\hline $\begin{array}{l}\text { C. glabrata } \\
\text { ATCC 200I }\end{array}$ & $16^{R}$ & $>64$ & 0.12 & 0.25 & 0.5 & 0.5 & 150 & $>600$ \\
\hline $\begin{array}{l}\text { C. glabrata } \\
\text { IAL-23 }\end{array}$ & $8^{\mathrm{SDD}}$ & $>64$ & 0.12 & 0.25 & 0.5 & I & 37.5 & $>600$ \\
\hline $\begin{array}{l}\text { C. krusei } \\
\text { ATCC } 6258\end{array}$ & $64^{\mathrm{R}}$ & $>64$ & 0.5 & 0.5 & 2 & 4 & 600 & $>600$ \\
\hline $\begin{array}{l}\text { C. krusei } \\
\text { IAL-30 }\end{array}$ & $64^{\mathrm{R}}$ & $>64$ & 0.25 & 0.5 & 1 & 1 & 600 & $>600$ \\
\hline $\begin{array}{l}\text { C. parapsilosis } \\
\text { ATCC } 22019\end{array}$ & 2 & 4 & 0.12 & 0.25 & 1 & 1 & 600 & $>600$ \\
\hline $\begin{array}{l}\text { C. parapsilosis } \\
\text { IAL-17 }\end{array}$ & $>64^{R}$ & $>64$ & 0.25 & 0.25 & 2 & 2 & 600 & $>600$ \\
\hline $\begin{array}{l}\text { C. tropicalis } \\
\text { ATCC } 200956\end{array}$ & $>64^{R}$ & $>64$ & $2^{R}$ & 2 & I & 2 & 600 & $>600$ \\
\hline $\begin{array}{l}\text { C. tropicalis } \\
\text { IAL-0I }\end{array}$ & 2 & 16 & 0.25 & 0.25 & 2 & 2 & 300 & $>600$ \\
\hline $\begin{array}{l}\text { C. neoformans } \\
\text { CAP59 }\end{array}$ & I & 32 & 0.03 & 0.03 & 1 & I & 1.56 & 1.56 \\
\hline $\begin{array}{l}\text { C. neoformans } \\
\text { H99 }\end{array}$ & 4 & 4 & 0.25 & 0.25 & 2 & 2 & 25 & 400 \\
\hline $\begin{array}{l}\text { C. gattii } \\
\text { ATCC } 56990\end{array}$ & 2 & 32 & 0.25 & 0.25 & 0.5 & 0.5 & 12.5 & 25 \\
\hline
\end{tabular}

Note: ${ }^{a}$ The assay was repeated and the results obtained here corroborated with previously published data. ${ }^{66}$

Abbreviations: MIC, minimum inhibitory concentration; MFC, minimum fungicidal concentration; R, resistant; SDD, susceptibility dose-dependent.

filamentation when compared to the untreated groups (Figure 5, Table S1); however, only the $200 \mathrm{mg} / \mathrm{Kg}$ dose showed these effects in the larvae infected with C. neoformans H99. Moreover, free MFS was less effective in decreasing these characteristics than MFS.Alg (Figure 5, Table S1).

\section{Discussion}

Sustained release systems have been widely used in the healthcare field as drug carriers. ${ }^{33}$ Here, we produced alginate nanoparticles as carriers for MFS by the external gelation/emulsification method. This is one of the most frequently used methods for the production of alginatebased delivery systems, and consists of two main steps: first, the alginate solution containing the drug is emulsified in an oil phase, forming an emulsion; and second, a crosslinking agent, most often calcium ions, is added to the emulsion, resulting in the gelation of the alginate droplets, followed by separation of the components from the emulsion. The advantage of this process is that the production of emulsions is relatively simple and can be scaled up for industrial use. ${ }^{22}$ 
A

B
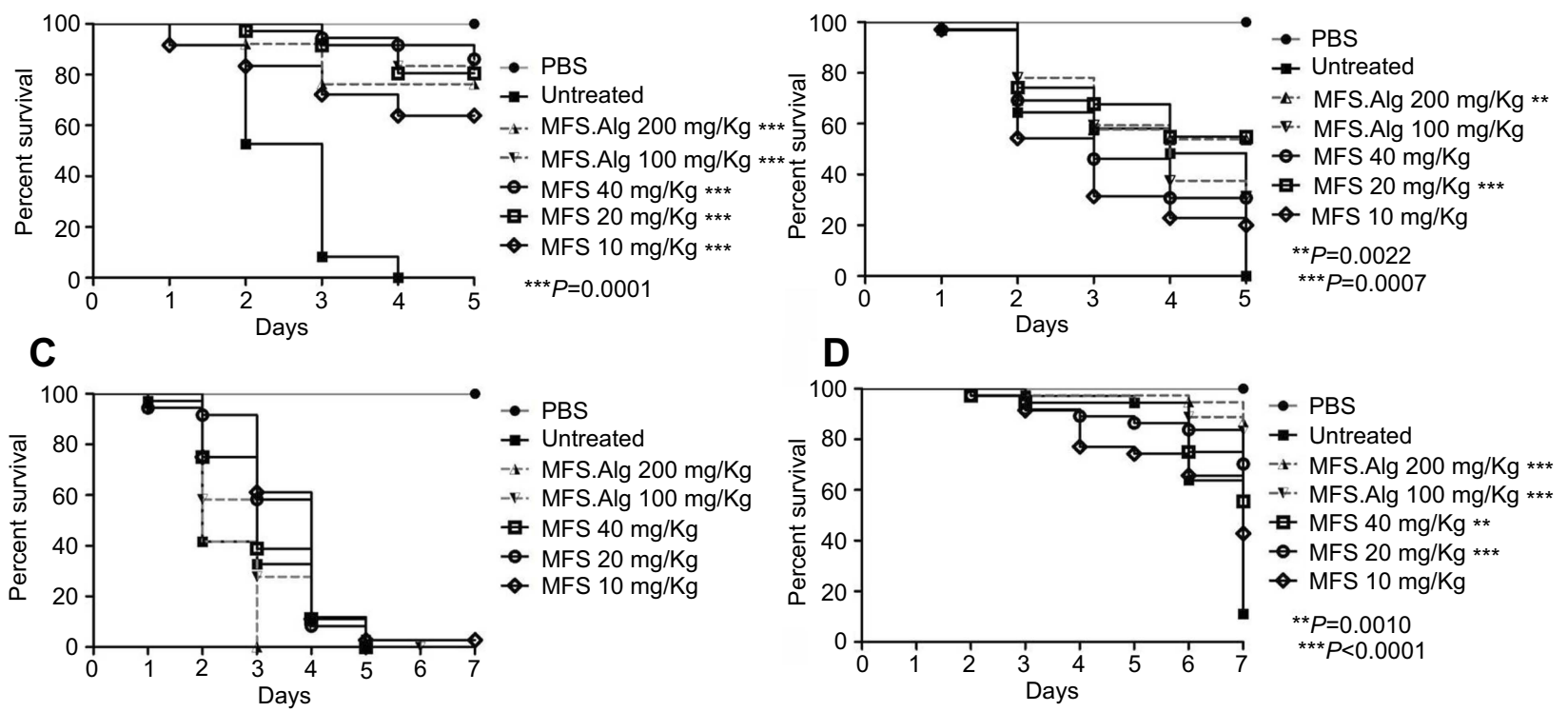

Figure 4 Survival curves of Galleria mellonella larvae infected with yeasts and treated 30 mins post-infection with free miltefosine (MFS) and miltefosine-loaded alginate nanoparticles (MFS.Alg). (A) Candida albicans SC5314, (B) Candida albicans IAL-40, (C) Cryptococcus neoformans H99, (D) Cryptococcus gattii ATCC 56990. P-values when compared with the untreated group.

Mean size, polydispersity index and zeta potential are extremely important parameters for drug delivery systems. The alginate nanoparticles loaded with MFS displayed a mean size of $279.1 \pm 56.7 \mathrm{~nm}$, which is well within the 200-1000 nm range reported for other alginate particles. ${ }^{34}$ To exemplify, alginate nanocarriers with mean sizes of 259 $\pm 27 \mathrm{~nm}$ and $419.60 \pm 0.28 \mathrm{~nm}$ were reported in previous studies, ${ }^{35,36}$ corroborating our data. Smaller particles can also be obtained, depending on the production process; for example, complexes of alginate hydrogel co-loaded with cisplatin and gold nanoparticles displayed a hydrodynamic diameter in the range of $20-250 \mathrm{~nm} .{ }^{37}$ The size displayed by MFS.Alg nanoparticles obtained here is favorable for mucosal and oral administration, since particles smaller than $500 \mathrm{~nm}$ have been described to be absorbed by transcellular/paracellular pathways across the intestinal mucosa. ${ }^{25,38}$ Conversely, it has also been demonstrated that nanoparticles are able to coat the mucosa, increasing the surface area of the contact with the drug and, consequently, the drug gradient concentration toward the blood. ${ }^{38,39}$ In our study, we did not attempt to discriminate the mechanisms.

In addition to size, nanoparticle charge also interferes with stability, behavior within the organism, and drug release. ${ }^{40}$ MFS.Alg presented a negative zeta potential $(-39.7 \pm 5.2 \mathrm{mV})$, which is consistent with the anionic nature of the polymer. ${ }^{41}$ Micro and nanoparticles with a negative zeta potential have been effectively used as carriers for antifungal drugs. ${ }^{35,42-44}$ Unlike positively charged nanoparticles that bind to cell membranes due to electrostatic interactions with the anionic membrane, the cellular uptake of negatively charged nanoparticles by the mucosa has been attributed, first, to the nonspecific adsorption process of the nanoparticles in the cell membrane and, second, to the formation of nanoparticle agglomerates. ${ }^{40,45}$

MFS encapsulation efficiency was approximately $80 \%$. Other studies using alginate carriers showed similar encapsulation efficiency values: microparticles with $\sim 80 \%$ nystatin, ${ }^{42}$ nanoparticles with $80-90 \%$ antituberculosis agents and $92-97 \%$ econazole. ${ }^{46}$ Small molecules have also been encapsulated with greater than $60 \%$ efficiency in nanocarriers produced with other polymers, such as PLGA, even when multiple production steps are used. ${ }^{47}$ The high percentage of drug encapsulation in the alginatebased nanoparticles makes these systems promising.

The sustained release of drugs promoted by the alginate-based nanocarriers is one of the attractive aspects of their use, since they might allow the drug to be constantly and slowly released into the body. The alginate polymer has a hydrophilic nature and drug release has been described to follow different mechanisms: water-soluble drugs seem to be released primarily by diffusion, whereas poorly water-soluble drugs, by matrix erosion. ${ }^{48}$ MFS has amphiphilic characteristics ${ }^{10}$ and its release from alginate 
Fungal burden
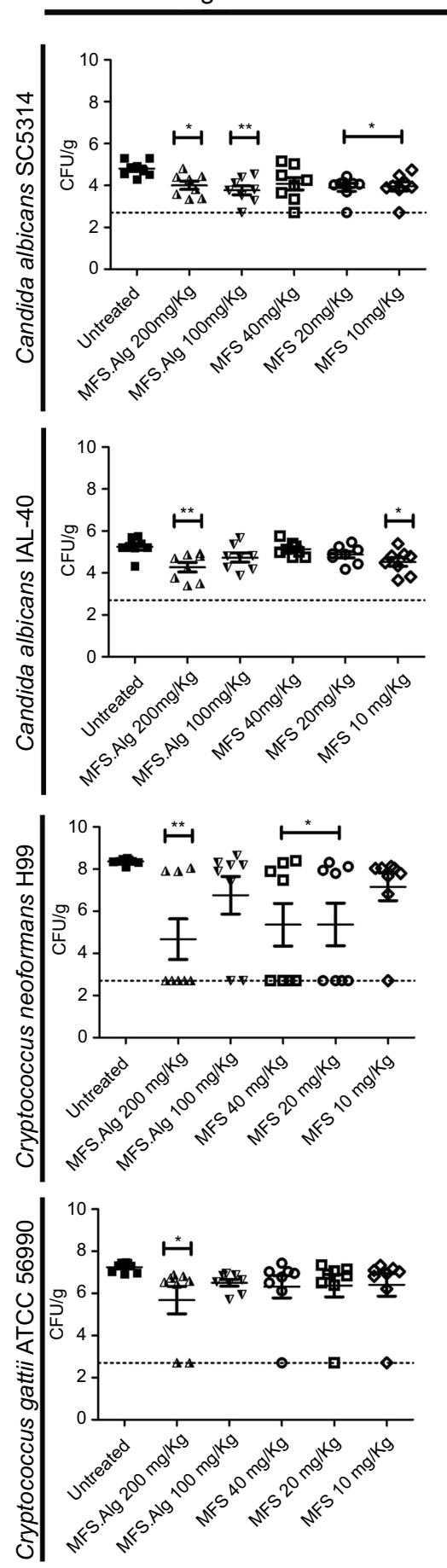

Histopathological analysis
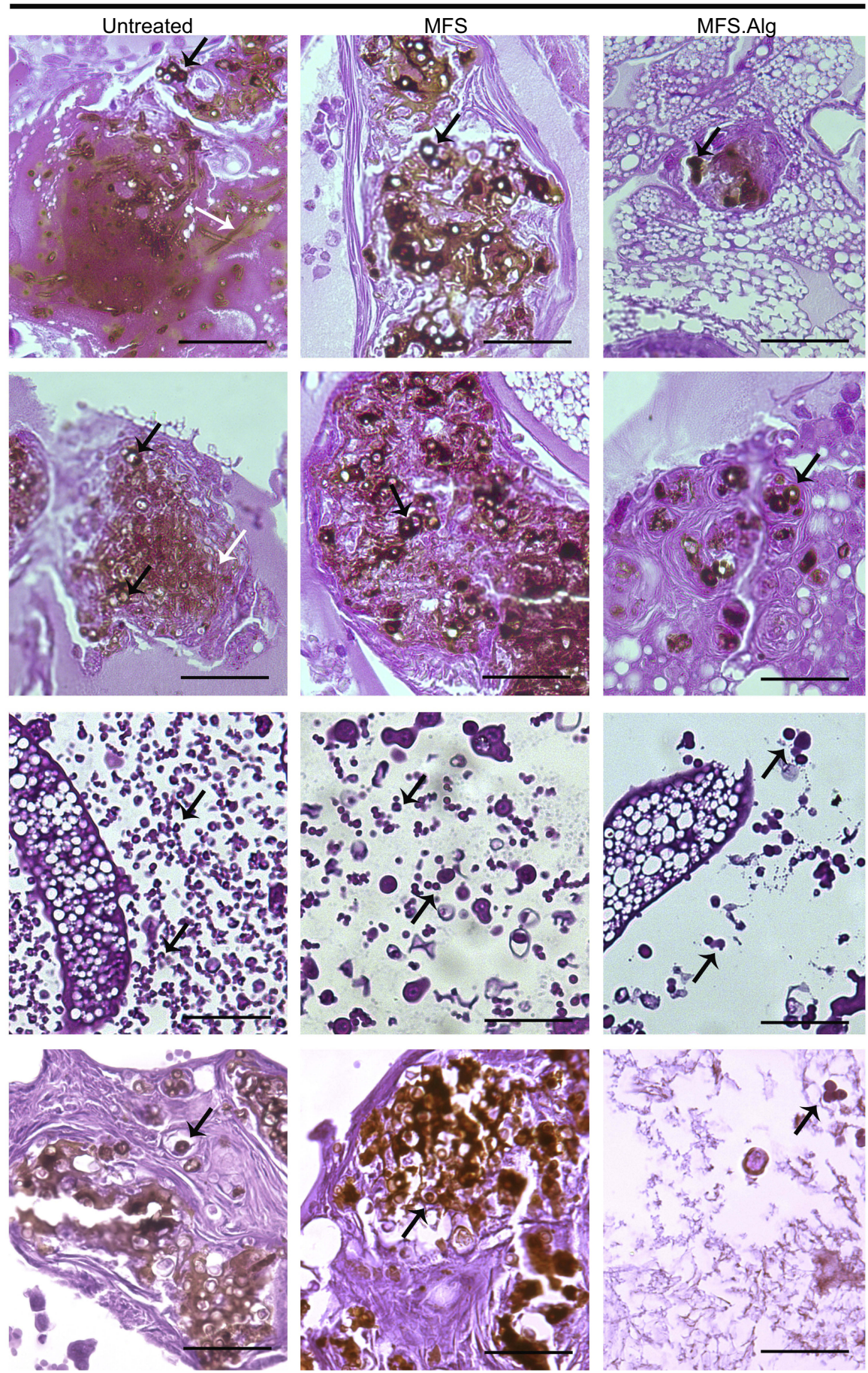

Figure 5 Fungal burden and histopathological analysis of Galleria mellonella larval tissue infected with yeasts and treated or not with free miltefosine (MFS) and miltefosineloaded alginate nanoparticles (MFS.Alg). Fungal burden data is represented by the mean \pm standard deviation. $* P<0.05$ and $* * P<0.01$ when compared with the untreated group (one-way ANOVA with Dunnet's post-test). Fungal burden, filamentation and dissemination of yeasts in Galleria mellonella larvae were analyzed by visualization of larval tissue histological sections of larvae untreated, treated with MFS $(40 \mathrm{mg} / \mathrm{kg})$, or treated with MFS.Alg $(200 \mathrm{mg} / \mathrm{kg})$. Black arrows indicate yeasts and white arrows indicate C. albicans filamentation. Bars: $50 \mu \mathrm{m}$.

nanoparticles might occur by diffusion and/or matrix erosion. Martín-Villena et al (2013) ${ }^{42}$ observed an initial stage with a burst of nystatin release from alginate microparticles, followed by a slower, sustained release phase.
A similar behavior was observed for MFS from the alginate nanoparticles, as a greater release of the MFS was observed at $6 \mathrm{~h}$, followed by a slower and steadier state for up to $24 \mathrm{~h}$. Because no chemical interactions were 
observed between the MFS and the formulation's constituents when analyzed by FTIR, no major formulationrelated hindrance for drug release is expected. The slow release observed may lead to a reduction in the dosing frequency, reducing fluctuations in plasma drug levels and the occurrence of adverse effects. ${ }^{49}$

It is known that MFS has a high toxicity associated with the kidneys and liver, teratogenic effect, pronounced hemolytic activity and gastrointestinal effects, such as nausea, vomiting, diarrhea, when used orally. ${ }^{19}$ We observed that MFS-loaded alginate nanoparticles (MFS.Alg) did not cause any hemolytic effect up to the highest concentration tested. In addition, MFS.Alg in G. mellonella larvae was significantly less toxic than free MFS. Moreover, it was observed that Nano.Alg do not present any toxic effects, corroborating literature reports. ${ }^{22,50}$ Our findings corroborate previous studies demonstrating that alginate-based carriers decrease drug toxic effects, including those of antifungal agents. ${ }^{23,35,51}$

The in vitro antifungal activity assay confirmed that MFS has an antifungal effect against Candida spp. and Cryptococcus spp. yeasts, with MIC values ranging from 0.5 to $2 \mu \mathrm{g} / \mathrm{mL}$, and fungicidal activity, especially effective on FLC-resistant strains; these data are in agreement with previous reports. ${ }^{13,52-55}$ An interesting aspect observed in the in vitro assay was the higher MIC values of MFS.Alg on Candida spp. when compared to Cryptococcus spp.; this can be explained by the slow and sustained release of MFS from alginate nanoparticles coupled with the faster replication time of Candida yeast, which makes the system less effective at inhibiting the fungal growth in vitro. Our data corroborate previous studies showing the possibility that antifungal nanocarriers may exhibit a lesser in vitro antifungal effect when compared to the free drug. ${ }^{56-58}$

Similar behavior was observed using the G. mellonella invertebrate model for fungal infection and treatment with free MFS and MFS.Alg, which exhibited analogous antifungal effects in doses of $20-40 \mathrm{mg} / \mathrm{kg}$ and $200 \mathrm{mg} / \mathrm{kg}$, respectively. Our alginate nanoparticles significantly reduced MFS toxicity in this model and were also effective at controlling the fungal infection in the in vivo larval model of G. mellonella, evidenced by the increase in larval survival rate and the decrease in fungal burden, filamentation and dissemination of yeasts in the larval tissue. Using murine models of fungal infection, previous studies have shown that, in addition to decreasing toxicological effects, the encapsulation may improve the drug's antifungal activity and contribute toward increasing the intervals between doses. ${ }^{56-58}$
Other studies have also demonstrated the use of nanocarriers based in PLGA-PEG and lipids for the reduction MFS toxicity. ${ }^{59-62}$ Polymeric carriers offer several advantages compared to the other systems, such as those formed by lipids, including the ease of production and scaling up, stability, and the fact that their physicochemical properties are amenable to reproduction after optimization of the production process. ${ }^{63}$ Furthermore, the use of natural polymers, such as alginate, is advantageous compared to synthetic polymers due to their abundance in nature, low processing cost, biocompatibility, biodegradability, water solubility, bioactivity and environmental safety. ${ }^{64,65}$

\section{Conclusion}

In conclusion, the results obtained here support the use of alginate polymer-based drug delivery systems as a carrier for MFS, decreasing its toxic effects when compared to the free drug, and controlling the fungal infection in the in vivo larval model of $G$. mellonella (Figure S4). Our results warrant further investigation of doses and regimens using MFS.Alg nanoparticles in mammalian models in order to support the use of MFS.Alg as an alternative to treat fungal diseases caused by Candida spp. and Cryptococcus spp. yeasts.

\section{Acknowledgments}

We are grateful to National Institute of Health Quality Control, Oswaldo Cruz Institute Foundation (Fiocruz, Rio de Janeiro/ RJ, Brazil), to Prof. Dr. Susana Frases Carvajal (Biophysics Institute Carlos Chagas Filho, Federal University of Rio de Janeiro, Rio de Janeiro/RJ, Brazil), and to Dr. Márcia de Souza Carvalho Melhem (Adolfo Lutz Institute, São Paulo/SP, Brazil) for kindly donating the yeasts samples. This work was supported by the Fundação de Amparo à Pesquisa do Estado de São Paulo (FAPESP - Brazil, grants grants 2015/ 07993-0, 2013/16617-7, and 2018/12149-2 ) and in part by the Conselho Nacional de Desenvolvimento Cientifico e Tecnológico (CNPq - Brasil) and Coordenação de Aperfeiçoamento de Pessoal de Nível Superior (CAPES Brazil, Finance Code 001). Part of the data described in this manuscript was previously presented at the 20th ISHAM congress (2018) as a poster presentation with interim findings. The poster's abstract was published in "Abstracts" in Medical Mycology, 2018;56:S1-S159: doi:10.1093/mmy/myy036.

\section{Author contributions}

All authors contributed to data analysis, drafting or revising the article, gave final approval of the version to be published, and agree to be accountable for all aspects of the work. 


\section{Disclosure}

Dr Cristina de Castro Spadari and Dr Fernanda Walt Mendes da Silva de Bastiani were participants of the fellowship program from Coordenação de Aperfeiçoamento de Pessoal de Nível Superior (CAPES). Dr Cristina de Castro Spadari reports a patent BR 1020170180115 pending; Dr Fernanda Walt Mendes da Silva de Bastiani reports a patent BR 102017 0180115 pending; Dr Luciana Biagini Lopes reports a patent BR 1020170180115 pending and Dr Kelly Ishida reports a patent BR 1020170180115 pending. Dr Luciana Biagini Lopes reports grants from São Paulo Research Foundation, during the conduct of the study and non-financial support from Abitech, outside the submitted work. The authors report no other conflicts of interest in this work.

\section{References}

1. Denning DW, Bromley MJ. How to bolster the antifungal pipeline. Science. 2015;347(6229):1414-1416. doi:10.1126/science.aaa6097

2. Ding H, Shen H, Huang Y, et al. BG40018: a promising drug candidate for the treatment of invasive fungal infections. Int J Clin Exp Med. 2017;10(10):14401-14407. doi:10.1109/AICI.2010.153

3. Brown GD, Denning DW, Gow NAR, Levitz SM, Netea MG, White TC. Hidden killers: human fungal infections. Sci Transl Med. 2012;4(165):1-9. doi:10.1126/scitranslmed.3004404

4. Spitzer M, Robbins N, Wright GD. Combinatorial strategies for combating invasive fungal infections. Virulence. 2017;8(2):169-185. doi:10.1080/21505594.2016.1196300

5. Odds FC, Brown AJP, Gow NAR. Antifungal agents: mechanisms of action. Trends Microbiol. 2003;11(6):272-279. doi:10.1016/S0966-842X (03)00117-3

6. Campoy S, Adrio JL. Antifungals. Biochem Pharmacol. 2017;133:86-96. doi:10.1016/j.bcp.2016.11.019

7. Fisher MC, Hawkins NJ, Sanglard D, Gurr SJ. Worldwide emergence of resistance to antifungal drugs challenges human health and food security. Science. 2018;360(6390):739-742. doi:10.1126/science.aap7999

8. Pianalto K, Alspaugh J. New horizons in antifungal therapy. J Fungi. 2016;2(4):26. doi:10.3390/jof2040026

9. Croft SL, Engel J. Miltefosine - discovery of the antileishmanial activity of phospholipid derivatives. Trans $\mathrm{R}$ Soc Trop Med Hyg 2006;100:1-5. doi:10.1016/j.trstmh.2006.03.009

10. Pachioni JDA, Magalhães JG, Lima EJC, et al. Alkylphospholipids a promising class of chemotherapeutic agents with a broad pharmacological spectrum. J Pharm Pharm Sci. 2013;16(5):742-759.

11. Sundar S, Chakravarty J. An update on pharmacotherapy for leishmaniasis. Expert Opin Pharmacother. 2015;16(2):237-252. doi:10.1517/14656566.2015.973850

12. Verhaar AP, Wildenberg ME, Peppelenbosch MP, Hommes DW, van den Brink GR. Repurposing miltefosine for the treatment of immune-mediated disease? J Pharmacol Exp Ther. 2014;350 (2):189-195. doi:10.1124/jpet.113.212654

13. de Castro Spadari C, Vila T, Rozental S, Ishida K. Miltefosine has a postantifungal effect and induces apoptosis in Cryptococcus yeasts. Antimicrob Agents Chemother. 2018;62(8):1-11. doi:10.1128/ AAC.00312-18

14. Brilhante RSN, Malaquias ADM, Caetano ÉP, et al. In vitro inhibitory effect of miltefosine against strains of Histoplasma capsulatum var. capsulatum and Sporothrix spp. Med Mycol. 2014;52 (3):320-325. doi:10.1093/mmy/myt027
15. Rossi DCP, de Castro Spadari C, Nosanchuk JD, Taborda CP, Ishida K. Miltefosine is fungicidal to Paracoccidioides spp. yeast cells but subinhibitory concentrations induce melanisation. Int $J$ Antimicrob Agents. 2017;49(4):465-471. doi:10.1016/j.ijantimicag.2016.12.020

16. Tong Z, Widmer F, Sorrell TC, et al. In vitro activities of miltefosine and two novel antifungal biscationic salts against a panel of 77 dermatophytes. Antimicrob Agents Chemother. 2007;51(6):2219-2222. doi:10.1128/ AAC.01382-06

17. Imbert S, Palous M, Meyer I, et al. In vitro combination of voriconazole and miltefosine against clinically relevant molds. Antimicrob Agents Chemother. 2014;58(11):6996-6998. doi:10.1128/AAC.03212-14

18. Vila TVM, Quintanilha NS, Rozental S. Miltefosine is effective against Candida albicans and Fusarium oxysporum nail biofilms in vitro. J Med Microbiol. 2015;64(11):1436-1449. doi:10.1099/ jmm.0.000175

19. Dorlo TPC, Balasegaram M, Beijnen JH, De Vries PJ. Miltefosine: a review of its pharmacology and therapeutic efficacy in the treatment of leishmaniasis. J Antimicrob Chemother. 2012;67:2576-2597. doi:10.1093/jac/dks275

20. Valenzuela-Oses JK, García MC, Feitosa VA, et al. Development and characterization of miltefosine-loaded polymeric micelles for cancer treatment. Mater Sci Eng C. 2017;81:327-333. doi:10.1016/j. msec.2017.07.040

21. Hosseini SM, Hosseini H, Mohammadifar MA, et al. Incorporation of essential oil in alginate microparticles by multiple emulsion/ionic gelation process. Int $J$ Biol Macromol. 2013;62:582-588. doi:10.1016/j.ijbiomac.2013.09.054

22. Paques JP, Van Der Linden E, Van Rijn CJM, Sagis LMC. Preparation methods of alginate nanoparticles. Adv Colloid Interface Sci. 2014;209:163-171. doi:10.1016/j.cis.2014.03.009

23. de Castro Spadari C, Lopes LB, Ishida K. Potential use of alginate-based carriers as antifungal delivery system. Front Microbiol. 2017;8:1-11. doi:10.3389/fmicb.2017.00097

24. Ishida K, Spadari C, Lopes LB, da Silva FWM. Formulação farmacêutica, processo para preparação da formulação farmacêutica, e uso da mesma. Patent INPI BR 102017018011 5; 2017.

25. Pandey R, Ahmad Z, Sharma S, Khuller GK. Nano-encapsulation of azole antifungals: potential applications to improve oral drug delivery. Int J Pharm. 2005;301:268-276. doi:10.1016/j.ijpharm.2005.05.027

26. Dorlo TPC, Eggelte TA, de Vries PJ, Beijnen JH. Characterization and identification of suspected counterfeit miltefosine capsules. Analyst. 2012;137(5):1265. doi:10.1039/c2an15641e

27. Li SP, Su ZR, Dong TTX, Tsim KWK. The fruiting body and its caterpillar host of Cordyceps sinensis show close resemblance in main constituents and anti-oxidation activity. Phytomedicine. 2002;9 (4):319-324. doi:10.1078/0944-7113-00134

28. Frenkel M, Mandelblat M, Alastruey-Izquierdo A, Mendlovic S, Semis R, Segal E. Pathogenicity of Candida albicans isolates from bloodstream and mucosal candidiasis assessed in mice and Galleria mellonella. J Mycol Med. 2016;26(1):1-8. doi:10.1016/j. mycmed.2015.12.006

29. CLSI. Reference method for broth dilution antifungal susceptibility testing of yeasts: approved standard - third edition. CLSI document M27-A3. Clin Lab Stand Inst. 2008;28(14)1-25.

30. CLSI. Reference method for broth dilution antifungal susceptibility testing of yeasts; fourth informational supplement. CLSI document M27-S4. 2012:28. doi:10.1094/PDIS-11-11-0999-PDN

31. Pfaller MA, Sheehan DJ, Rex JH. Determination of fungicidal activities against yeasts and molds: lessons learned from bactericidal testing and the need for standardization. Clin Microbiol Rev. 2004;17(2):268-280. doi:10.1128/CMR.17.2.268-280.2004

32. Quintella LP, Lambert Passos SR, Francesconi Do Vale AC, et al. Histopathology of cutaneous sporotrichosis in Rio de Janeiro: a series of 119 consecutive cases. J Cutan Pathol. 2011;38(1):25-32. doi:10.1111/j.1600-0560.2010.01626.x 
33. Mojtaba Salouti, Azam Ahangari. Application of Nanotechnology in Drug Delivery. In: Sezer AD, editor. Nanoparticle based drug delivery systems for treatment of infectious diseases. London: IntechOpen; 2014.

34. Paques JP, Van der Linden E, Van Rijn CJM, Sagis LMC. Alginate submicron beads prepared through w/o emulsification and gelation with $\mathrm{CaCl}_{2}$ nanoparticles. Food Hydrocoll. 2013;31(2):428-434. doi:10.1016/j.foodhyd.2012.11.012

35. Sangeetha S, Venkatesh DN, Adhiyaman R, Santhi K, Suresh B. Formulation of sodium alginate nanospheres containing amphotericin B for the treatment of systemic candidiasis. Trop J Pharm Res. 2007;6:653-659. doi:10.4314/tjpr.v6i1.14643

36. Tachaprutinun A, Pan-In P, Wanichwecharungruang S. Mucosa-plate for direct evaluation of mucoadhesion of drug carriers. Int J Pharm. 2013;441(1-2):801-808. doi:10.1016/j.ijpharm.2012.12.028

37. Keshavarz M, Moloudi K, Paydar R, et al. Alginate hydrogel co-loaded with cisplatin and gold nanoparticles for computed tomography image-guided chemotherapy. J Biomater Appl. 2018;33 (2):161-169. doi: $10.1177 / 0885328218782355$

38. Jiao Y, Ubrich N, Marchand-Arvier M, et al. In vitro and in vivo evaluation of oral heparin-loaded polymeric nanoparticles in rabbits. Circulation. 2002;105(2):230-235. doi:10.1161/hc0202.101988

39. Laksitorini M, Prasasty VD, Kiptoo PK, Siahaan TJ. Pathways and progress in improving drug delivery through the intestinal mucosa and blood-brain barriers. Ther Deliv. 2014;5(10):1143-1163. doi: $10.4155 /$ tde. 14.67

40. Honary S, Zahir F. Effect of zeta potential on the properties of nanodrug delivery systems - a review (part 1). Trop J Pharm Res. 2013;12 (2):265-273. doi:10.4314/tjpr.v12i2.19

41. Gupta PK, Jaiswal AK, Asthana S, et al. Self assembled ionically sodium alginate cross-linked amphotericin b encapsulated glycol chitosan stearate nanoparticles: applicability in better chemotherapy and non-toxic delivery in visceral leishmaniasis. Pharm Res. 2015;32 (5):1727-1740. doi:10.1007/s11095-014-1571-4

42. Martín-Villena MJ, Fernández-Campos F, Calpena-Campmany AC, Bozal-de Febrer N, Ruiz-Martínez MA, Clares-Naveros B. Novel microparticulate systems for the vaginal delivery of nystatin: development and characterization. Carbohydr Polym. 2013;94(1):1-11. doi:10.1016/j.carbpol.2013.01.005

43. Martín MJ, Calpena AC, Fernández F, Mallandrich M, Gálvez P, Clares B. Development of alginate microspheres as nystatin carriers for oral mucosa drug delivery. Carbohydr Polym. 2015;117:140-149. doi:10.1016/j.carbpol.2014.09.032

44. Reis CP, Roque LV, Baptista M, Rijo P. Innovative formulation of nystatin particulate systems in toothpaste for candidiasis treatment. Pharm Dev Technol. 2015;7450:1-6. doi:10.3109/10837450.2014.999783

45. Wilhelm C, Billotey C, Roger J, Pons JN, Bacri JC, Gazeau F. Intracellular uptake of anionic superparamagnetic nanoparticles as a function of their surface coating. Biomaterials. 2003;24 (6):1001-1011. doi:10.1016/S0142-9612(02)00440-4

46. Ahmad Z, Sharma S, Khuller GK. Chemotherapeutic evaluation of alginate nanoparticle-encapsulated azole antifungal and antitubercular drugs against murine tuberculosis. Nanomed Nanotechnol Biol Med. 2007;3(3):239-243. doi:10.1016/j.nano.2007.05.001

47. Shakeri-Zadeh A, Shiran MB, Khoee S, Sharifi AM, Ghaznavi H, Khoei S. A new magnetic nanocapsule containing 5-fluorouracil: in vivo drug release, anti-tumor, and pro-apoptotic effects on CT26 cells allograft model. J Biomater Appl. 2014;29(4):548-556. doi: $10.1177 / 0885328214536940$

48. Sosnik A. Alginate particles as platform for drug delivery by the oral route: state-of-the-art. ISRN Pharm. 2014;2014:1-17. doi:10.1155/ 2014/926157

49. Jain KK. Drug Delivery System. Vol. 1141. (Jain KK, editor). New York, NY: Springer New York; 2014. doi:10.1007/978-1-4939-0363-4
50. Cardoso M, Costa R, Mano J. Marine origin polysaccharides in drug delivery systems. Mar Drugs. 2016;14(2):34. doi:10.3390/md14020034

51. Kumar A, Chen F, Mozhi A, et al. Innovative pharmaceutical development based on unique properties of nanoscale delivery formulation. Nanoscale. 2013;5(18):8307. doi:10.1039/c3nr01525d

52. Vila TVM, Chaturvedi AK, Rozental S, Lopez-Ribot JL. In vitro activity of miltefosine against Candida albicans under planktonic and biofilm growth conditions and in vivo efficacy in a murine model of oral candidiasis. Antimicrob Agents Chemother. 2015;59 (12):7611-7620. doi:10.1128/AAC.01890-15

53. Widmer F, Wright LC, Obando D, et al. Hexadecylphosphocholine (miltefosine) has broad-spectrum fungicidal activity and is efficacious in a mouse model of cryptococcosis. Antimicrob Agents Chemother. 2006;50(2):414-421. doi:10.1128/AAC.50.2.414-421.2006

54. Ravu RR, Chen YL, Jacob MR, et al. Synthesis and antifungal activities of miltefosine analogs. Bioorg Med Chem Lett. 2013;23 (17):4828-4831. doi:10.1016/j.bmcl.2013.06.096

55. Vila T, Ishida K, Seabra SH, Rozental S. Miltefosine inhibits Candida albicans and non-albicans Candida spp. biofilms and impairs the dispersion of infectious cells. Int $J$ Antimicrob Agents. 2016;48(5):512-520. doi:10.1016/j.ijantimicag.2016.07.022

56. Khan AA, Jabeen M, Alanazi AM, Khan AA. Antifungal efficacy of amphotericin B encapsulated fibrin microsphere for treating Cryptococcus neoformans infection in Swiss albino mice. Brazilian J Infect Dis. 2016;20(4):342-348. doi:10.1016/j.bjid.2016.04.006

57. Saldanha CA, Garcia MP, Iocca DC, et al. Antifungal activity of amphotericin B conjugated to nanosized magnetite in the treatment of paracoccidioidomycosis. Vinetz JM, ed. PLoS Negl Trop Dis. 2016;10(6):e0004754. doi:10.1371/journal.pntd.0004754

58. Tan TRM, Hoi KM, Zhang P, Ng SK. Characterization of a polyethylene glycol-amphotericin $\mathrm{B}$ conjugate loaded with free AMB for improved antifungal efficacy. PLoS One. 2016;11 (3):1-18. doi:10.1371/journal.pone.0152112

59. Eissa MM, El-Moslemany RM, Ramadan AA, et al. Miltefosine lipid nanocapsules for single dose oral treatment of Schistosomiasis Mansoni: a preclinical study. PLoS One. 2015;10(11):e0141788. doi:10.1371/journal.pone. 0141788

60. El-Moslemany RM, Eissa MM, Ramadan AA, El-Khordagui LK, ElAzzouni MZ. Miltefosine lipid nanocapsules: intersection of drug repurposing and nanotechnology for single dose oral treatment of pre-patent schistosomiasis mansoni. Acta Trop. 2016;159:142-148. doi:10.1016/j.actatropica.2016.03.038

61. Kumar R, Sahoo GC, Pandey K, et al. Development of PLGA-PEG encapsulated miltefosine based drug delivery system against visceral leishmaniasis. Mater Sci Eng C. 2016;59:748-753. doi:10.1016/j. msec.2015.10.083

62. da Gama Bitencourt JJ, Pazin WM, Ito AS, et al. Miltefosine-loaded lipid nanoparticles: improving miltefosine stability and reducing its hemolytic potential toward erythtocytes and its cytotoxic effect on macrophages. Biophys Chem. 2016;217:20-31. doi:10.1016/j.bpc.2016.07.005

63. Voltan AR, Quindós G, Alarcón KPM, Fusco-Almeida AM, MendesGiannini MJS, Chorilli M. Fungal diseases : could nanostructured drug delivery systems be a novel paradigm for therapy? Int J Nanomedicine. 2016;11:3715-3730. doi:10.2147/IJN.S93105

64. Debele TA, Mekuria SL, Tsai H-C. Polysaccharide based nanogels in the drug delivery system: application as the carrier of pharmaceutical agents. Mater Sci Eng C. 2016. doi:10.1016/j.msec.2016.05.121

65. Sundar S, Kundu J, Kundu SC. Biopolymeric nanoparticles. Sci Technol Adv Mater. 2010;11(1):014104. doi:10.1088/1468-6996/11/1/014104

66. Muñoz JE, Rossi DCP, Ishida K, et al. Antifungal activity of the biphosphinic cyclopalladate $\mathrm{C} 7 \mathrm{a}$ against Candida albicans yeast forms In vitro and In vivo. Front Microbiol. 2017;8:771. doi:10.3389/fmicb.2017.00771 


\section{Publish your work in this journal}

The International Journal of Nanomedicine is an international, peerreviewed journal focusing on the application of nanotechnology in diagnostics, therapeutics, and drug delivery systems throughout the biomedical field. This journal is indexed on PubMed Central, MedLine, CAS, SciSearch ${ }^{\mathbb{R}}$, Current Contents ${ }^{\mathbb{B}} /$ Clinical Medicine,
Journal Citation Reports/Science Edition, EMBase, Scopus and the Elsevier Bibliographic databases. The manuscript management system is completely online and includes a very quick and fair peer-review system, which is all easy to use. Visit http://www.dovepress.com/ testimonials.php to read real quotes from published authors. 